\title{
La mobilité des hommes et des idées : la Louisiane espagnole face aux mouvements révolutionnaires entre 1790 et 1803
}

\section{Soizic Croguennec}

\section{(2) OpenEdition}

\section{Journals}

Édition électronique

URL : https://journals.openedition.org/geohist/1289

DOI : 10.4000/geohist.1289

ISSN : 2264-2617

Éditeur

Association française de la Revue de géographie historique

Référence électronique

Soizic Croguennec, «La mobilité des hommes et des idées : la Louisiane espagnole face aux mouvements révolutionnaires entre 1790 et 1803 », Revue de géographie historique [En ligne], 13 | 2018 mis en ligne le 20 novembre 2018, consulté le 12 juin 2021. URL : http://journals.openedition.org/ geohist/1289; DOI : https://doi.org/10.4000/geohist.1289

Ce document a été généré automatiquement le 12 juin 2021.

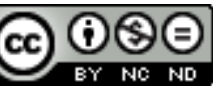

Ce(tte) œuvre est mise à disposition selon les termes de la Licence Creative Commons Attribution -

Pas d'Utilisation Commerciale - Pas de Modification 4.0 International. 


\title{
La mobilité des hommes et des
} idées : la Louisiane espagnole face aux mouvements révolutionnaires entre 1790 et 1803

\author{
Soizic Croguennec
}

\section{Introduction}

1 En acceptant la Louisiane à l'issue de la guerre de Sept ans, la Couronne espagnole reçoit un immense territoire perçu davantage comme une charge que comme un atout. L'historien Alfredo Jiménez évoque un véritable « cadeau empoisonné » (Jiménez, 2006) de la part de Louis XV qui confie ainsi à son cousin une colonie peu développée et coûteuse au terme d'un gigantesque jeu de chaises musicales qui redessine le visage de l'Amérique du Nord. La France laisse le Canada à la Grande Bretagne pour récupérer ses possessions antillaises tandis que l'Espagne abandonne la Floride pour reprendre Cuba. La cession de la Louisiane par Louis XV est à la fois une récompense pour la solidarité dynastique affichée par son cousin Charles III et une forme de compensation pour la perte des anciennes colonies floridiennes (Jiménez, 2006). En réalité, pour les autorités espagnoles, la Louisiane possède avant tout un intérêt géopolitique comme le souligne l'ambassadeur Jerónimo Grimaldi lors des négociations du Traité de Paris lorsqu'il accepte les conditions au nom de son roi «bien qu'il sache parfaitement qu'[ils] ne [faisaient] qu'acquérir une charge annuelle de 300000 piastres, en échange de l'utilité négative et douteuse de posséder un pays pour que d'autres ne le possèdent pas » (Andreu Ocariz, 1975). La perte de la Floride affaiblit le contrôle du golfe du Mexique par l'Espagne et rapproche la Grande-Bretagne des richesses convoitées de NouvelleEspagne. Dans cette nouvelle configuration, la Louisiane doit servir de barrière protégeant la Nouvelle-Espagne et ses ressources argentifères des appétits de la Couronne et des aventuriers britanniques (Narrett, 2014). 


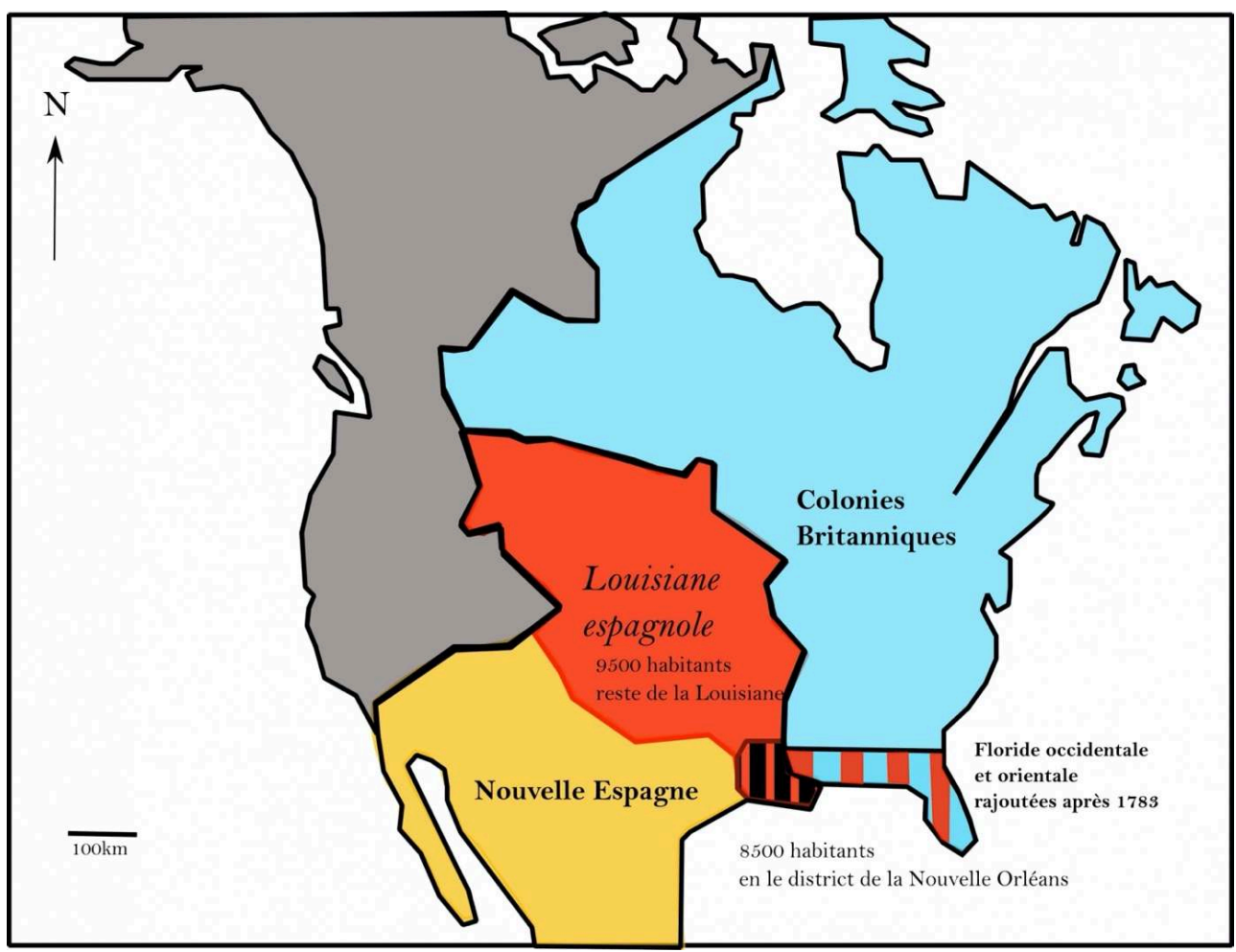

En outre, même si les colonies françaises au Canada et en Louisiane étaient peu peuplées, la reconfiguration géopolitique du sous-continent décidée dans les confins des palais européens affecte la population locale durement. Les Français de Louisiane doivent ainsi accepter l'autorité d'un nouveau roi, le roi d'Espagne, et s'adapter à un nouveau système économique, fiscal et administratif. De ce fait, le sentiment d'un abandon matériel et symbolique voire d'une véritable trahison de la part de Louis $\mathrm{XV}$ vis-à-vis de ses sujets louisianais est particulièrement marqué. La révolte de la population française en 1768 contre le premier gouverneur Antonio de Ulloa arrivé tardivement en 1766 est ainsi révélatrice à la fois de l'attachement de la population locale à son ancien roi et du rejet suscité par la domination d'une puissance perçue comme archaïque, despotique et obscurantiste, qui « fait trembler de peur tout homme né libre $»^{1}$. Au-delà de la dimension politique, la révolte est également alimentée par le refus d'une intégration contrainte au monopole commercial espagnol qui détruirait les liens et réseaux tissés depuis le début du XVIII siècle avec les Antilles et les ports français (Havard et Vidal, 2008). L'intervention brutale d'Alejandro O'Reilly met fin à la révolte et pose les bases d'une administration coloniale associant les élites françaises au gouvernement et accordant le maintien du commerce avec l'ancienne métropole (Hoffman, 1992).

3 Un tel système peut être vu comme un véritable laboratoire colonial qui place la Louisiane désormais espagnole au cœur du système atlantique et caribéen comme le soulignent Cécile Vidal (Vidal, 2014) et Shannon Lee Dawdy (Dawdy, 2007). "Carrefour", «nexus" mais aussi "confins", "frontière" les expressions ne manquent pas pour décrire la position à la fois centrale et marginale de la Louisiane entre empire espagnol, français et britannique puis étatsunien. Plaque tournante qui relie l'intérieur du continent via la vallée du Mississippi au golfe du Mexique, la 
Louisiane, et plus particulièrement la Nouvelle-Orléans, se trouve ainsi au cœur des flux transatlantiques, caribéens et nord-américains. Dans le même temps, le territoire louisianais est aussi une région de confins, peu contrôlée par les différents pouvoirs coloniaux en présence et donc favorable aux circulations illicites de tous genres - fuite d'esclaves, désertions de soldats, contrebande.

Document 2 : La Louisiane espagnole, « carrefour du monde atlantique » (Cécile Vidal, 2014)

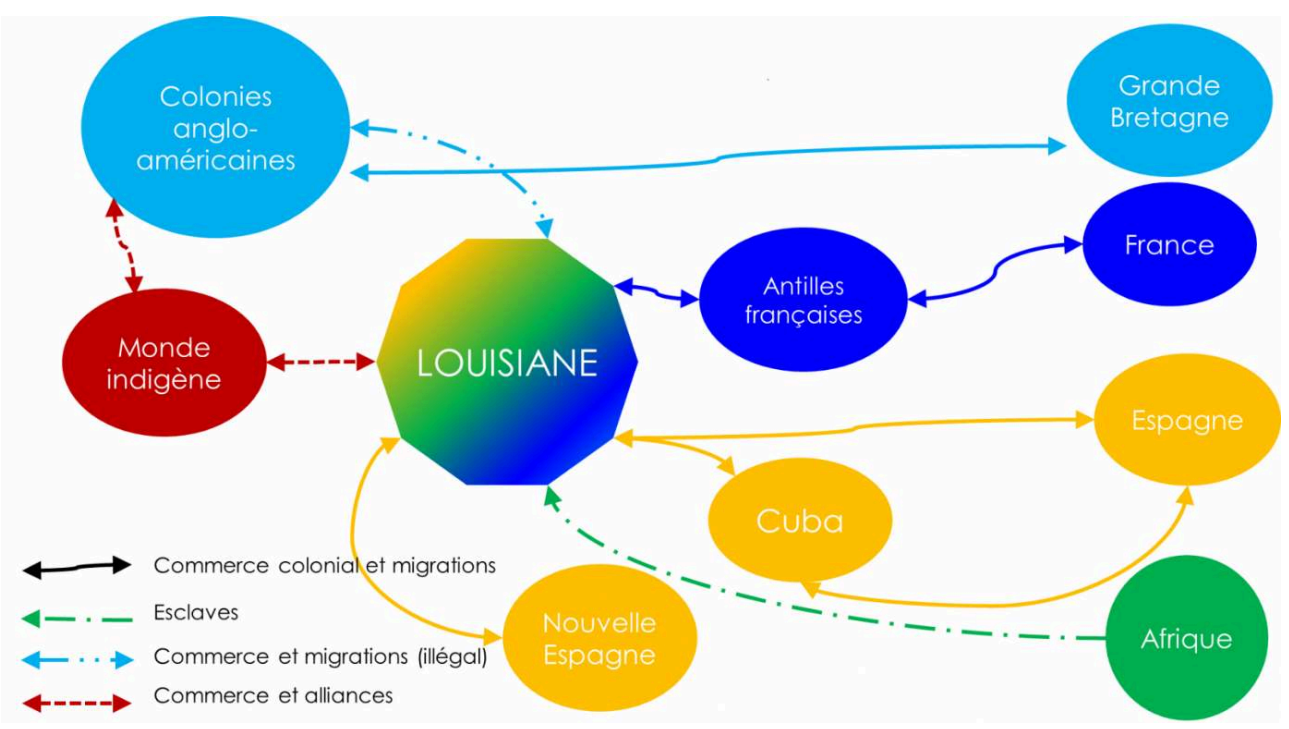

Dans le contexte de la fin du XVIII ${ }^{\mathrm{e}}$ siècle, ces dynamiques de circulation tendent à exacerber les tensions révolutionnaires comme contre-révolutionnaires (Mongey, 2009). Les autorités espagnoles sont en effet confrontées à la circulation des idées et des livres prohibés du fait de la connexion commerciale de la Louisiane à la France. De glacis protecteur sur le plan militaire, la région devient une véritable porte d'entrée par laquelle les idéaux révolutionnaires peuvent atteindre le cœur de l'Empire espagnol, en Nouvelle-Espagne et au-delà. A l'opposé, la Louisiane sous contrôle du roi d'Espagne fait également figure de refuge et attire ainsi d'importants flux migratoires originaires de Saint-Domingue au cours de la première décennie du XIX $\mathrm{X}^{\mathrm{e}}$ siècle (Dessens, 2007). À partir de l'analyse de la documentation espagnole, et plus particulièrement les fonds Inquisición des Archivos Generales de la Nación (AGN) de Mexico et Pasaportes de l'Archivo General de Indias (AGI) de Séville, il est possible de retracer ces dynamiques en reconstituant des itinéraires qui permettent d'apprécier la manière dont les événements révolutionnaires affectent les stratégies individuelles et collectives par un jeu de dominos au centre duquel se trouve la Louisiane espagnole.

\section{Circulations transatlantiques et idées révolutionnaires}

Pour la Couronne espagnole, la Louisiane est avant tout considérée pour son importance géostratégique : elle sert de glacis protecteur censé mettre la NouvelleEspagne à l'abri des convoitises anglo-américaines tout en maintenant la présence espagnole au nord du Golfe du Mexique. De ce point de vue, l'axe du Mississippi revêt un rôle essentiel pour pénétrer au cœur du continent américain et bloquer l'expansion des colonies anglaises puis des États-Unis vers l'Ouest. Une telle position de repli et de 
contrôle est en contradiction totale avec la vision anglo-américaine de la liberté de circulation des biens et des personnes sur le continent en général et sur le Mississippi en particulier. De fait, malgré la volonté de contrôle des autorités espagnoles, la Louisiane est particulièrement vulnérable face aux différents mouvements de contrebande générés par la proximité du monde anglo-américain d'une part et par les liens commerciaux maintenus avec la France et les Antilles françaises à la suite des concessions d'Alejandro O'Reilly d'autre part. En effet, la politique espagnole en Louisiane se distingue par un pragmatisme permanent pour s'attacher les fidélités des acteurs locaux. Sylvia Hilton évoque ainsi l'idée d'un « empire négocié » (Hilton, 2014).

\section{A. Le paradoxe louisianais : un « glacis protecteur perméable »}

6 Ce paradoxe est notamment visible lorsque l'on se penche sur la documentation inquisitoriale de la toute fin du XVIII siècle. Conçue comme un tribunal religieux chargé de la lutte contre l'hétérodoxie religieuse sous toutes ses formes, l'Inquisition espagnole est devenue à la fin de la période moderne un instrument de contrôle social et politique avant tout. Dans le contexte des "révolutions atlantiques " (Godechot, 1986 ; Jourdan, 2006), l'institution constitue ainsi pour le pouvoir royal des Bourbons d'Espagne le principal rempart contre les idées perçues comme «impies et séditieuses ». Or, la position de la Louisiane espagnole comme "carrefour du monde atlantique " pour reprendre l'expression de Cécile Vidal la place au cœur des flux licites et illicites des hommes comme des marchandises, et donc des idées. Un premier axe " américain» peut être identifié entre les États-Unis et la Nouvelle-Espagne via la Nouvelle-Orléans. C'est ce que montre l'itinéraire du livre interdit El Desengaño del Hombre publié par Santiago Felipe Puglia à Philadelphie en 1794. L'ouvrage, qui critique vertement le principe de monarchie absolue de droit divin, s'attire les foudres de la censure. On retrouve là des mécanismes similaires à l'interdiction et saisie de la traduction de la Déclaration des Droits de l'Homme et du Citoyen par Antonio Nariño la même année à Bogotá (Lomné, 2011). Avertis par le baron de Carondelet des intentions de Puglia de diffuser son livre en territoire espagnol à partir de Philadelphie, le vice-roi et l'Inquisition de Mexico prennent des mesures pour s'opposer à la diffusion du livre interdit en Nouvelle-Espagne :

7 "Le capitaine de Louisiane et de Floride, dans la lettre du 26 septembre de cette année a rendu compte de la remise au vice-roi de Nouvelle-Espagne d'un livre, imprimé à Philadelphie, en espagnol, intitulé Desengaño del hombre, par l'auteur don Felipe Santiago Puglia, maître de cette même langue, dont l'oeuvre est parsemée de propositions vénéneuses et nuisibles. Et comme il y a des índices selon lesquels [l'auteur] essaie d'introduire des exemplaires dans le dit royaume de NouvelleEspagne via la Louisiane, le gouvernement a pris les dispositions suivantes pour l'empêcher $»^{2}$.

8 A travers cet itinéraire, ce sont les liens tissés entre les ports étatsuniens et la NouvelleOrléans que l'on retrouve. D'abord timides, l'intensité de ces liens s'est renforcée dans les dernières années du XVIIIe siècle à mesure que les réseaux marchands angloaméricains se sont déployés vers l'Ouest et le long du bassin du Mississippi jusqu'à la Nouvelle-Orléans. Dans cet espace, la liberté de circulation est un principe fondateur pour les anglo-américains. Dans le procès qui l'oppose au Français Joseph Leblanc, le marchand Arthur Morgan insiste sur l'importance de cette liberté de circulation entre 
la Nouvelle-Orléans et les États-Unis. Il déclare ainsi "qu'il est mal de le retenir [à la Nouvelle-Orléans] car non seulement cela [le] prive de la liberté dont dispose tout individu pour voyager et mener ses affaires mais aussi parce [il] souffrira également d'un grand préjudice pour cela ${ }^{3} »$. Les maisons de commerce de Baltimore, New York ou Philadelphie s'attachent donc à fonder des comptoirs à la Nouvelle-Orléans (Narrett, 2014) pour renforcer leur position à l'embouchure du Mississippi et capter ainsi l'argent de Nouvelle-Espagne qui irrigue les marchés louisianais par l'intermédiaire du situado (Marichal et Souto, 2012). Ce sont ces réseaux que mobilise Felipe Puglia lorsqu'il veut diffuser sa critique de la monarchie absolue en terre hispanique, Philadelphie jouant le rôle d'une véritable base arrière des mouvements révolutionnaires touchant le monde hispano-américain à partir des années 1790 et plus encore après 1810 (Terrien, 2015).

Au-delà des réseaux entre Etats-Unis et Nouvelle-Orléans, les idées "séditieuses » menacent de toucher la Nouvelle-Espagne par les anciens circuits antillais et français. C'est ainsi que les autorités du fort de Natchitoches, sur la frontière avec le Texas, en face de Los Adaes, saisissent des livres de Voltaire en 1799 avant qu'ils ne pénètrent en Nouvelle-Espagne :

«Dossier instruit contre le capitaine Bernardo Northolan, milice du poste de Natchitoches, province de Louisiane, pour avoir introduit deux deux tomes de l'oeuvre de Voltaire "

10 La population française de Louisiane est imprégnée par les Lumières et maintient des liens culturels forts avec l'ancienne métropole (Vidal, 2014), ce qui n'est pas acceptable pour une couronne espagnole qui s'arcboute pour étouffer les velléités révolutionnaires de ses populations en péninsule comme en Amérique et conserver la plénitude de son empire. Dans ce contexte, la libre-circulation de marchands français originaires de la Nouvelle-Orléans et acquis aux idées révolutionnaires dans le reste des possessions espagnoles peut constituer un véritable danger. C'est ce que l'on peut voir à travers l'itinéraire de Jean Langouran.

11 B. Le cas de Jean Langouran, de Bordeaux à Tegucigalpa

12 Jean Langouran est un marchand originaire de Bordeaux et installé à la NouvelleOrléans où il s'est marié[9]. Comme capitaine français du navire la Luisa, il peut tout d'abord s'appuyer sur les réseaux commerciaux atlantiques entre la Louisiane et le port de Bordeaux. Ces liens lui permettent de développer son activité mais aussi le mettent en contact direct avec les événements et idées révolutionnaires auxquels il se montre favorable. Mais comme marchand de la Nouvelle-Orléans, il appartient également à la catégorie des vecinos y del comercio ainsi que sujet du roi d'Espagne, ce qui lui donne accès aux réseaux commerciaux de l'Empire espagnol grâce à un passeport délivré par le gouverneur de la Louisiane Esteban Miro. C'est ainsi qu'on le retrouve en 1794 dans la capitainerie générale du Guatemala à Tegucigalpa accusé de défendre et répandre des idées séditieuses. L'acte d'accusation formulé par l'Inquisition de Mexico affirme ainsi qu'il a "accumulé des productions séditieuses contre le roi de France en faveur de l'Assemblée Nationale et certains points de religion ».

13 La formulation est particulièrement intéressante dans la mesure où Langouran n'est pas accusé de critiquer la monarchie espagnole - comme c'est le cas du livre de Felipe Puglia. Ici, ce sont ses opinions au sujet des événements français et ses prises de position sur le roi Louis XVI et les actions de l'Assemblée Législative puis de la Convention entre 1792 et 1793. La destitution de Louis XVI puis sa condamnation à 
mort a véritablement eu l'effet d'un coup de tonnerre pour les monarchies européennes qui se sentent toutes menacées par une éventuelle contagion révolutionnaire qui viendrait les renverser à leur tour. Tout comme le «spectre de la Révolution Noire» frappe les sociétés esclavagistes du bassin caribéen au point de prendre des mesures pour se prémunir des récits rapportés par les réfugiés de SaintDomingue (Gómez, 2017), le spectre du roi condamné à mort plane sur les consciences en Europe comme en Amérique. En s'attaquant à Jean Langouran qui s'enthousiasme pour la Convention jusqu'à Tegucigalpa, l'Inquisition tente de limiter la diffusion d'idées qui pourraient trouver un écho favorable auprès des populations locales.

Les questions politiques ne sont pas les seules qui rendent Langouran dangereux aux yeux de l'Inquisition. Qualifié de médecin, il est aussi accusé de libertinage. Ses pratiques religieuses sont ainsi rigoureusement examinées - assistance à la messe, communion, réalité de son mariage à la Nouvelle-Orléans. Le cas de Langouran permet de mettre le doigt sur le fossé religieux qui sépare la population française des autorités espagnoles. Le processus de sécularisation et de déprise religieuse de la deuxième moitié du XVIII ${ }^{e}$ siècle touche la Louisiane à l'image de son ancienne métropole. À l'inverse, l'Espagne se crispe au cours de la même période sur la défense du catholicisme tridentin. Avec l'intégration de la Louisiane à l'empire colonial espagnol, deux dynamiques religieuses inverses se retrouvent ainsi confrontées directement. Le comportement et les paroles d'un marchand français comme Langouran au cœur de l'empire focalisent donc l'attention des autorités et des populations qui le côtoient au quotidien. Il est ainsi intéressant que Langouran est dénoncé par ses voisins pour ses propos sur la religion et la Révolution française. Les mécanismes des dénonciations auprès de l'Inquisition sont complexes - il n'est pas rare de voir des individus ayant recours à un guérisseur le dénoncer après le rituel de manière préemptive - et il est donc difficile de cerner les motivations des dénonciateurs. Le rejet catégorique des idées portées par Langouran peut être une hypothèse, tout comme la méfiance à l'égard d'un étranger ou encore la volonté de se protéger soi-même de la curiosité de l'Inquisition. De fait, Langouran est un homme qui est vu comme un perturbateur de l'ordre public sur le plan politique comme religieux, un élément étranger qui a pu s'introduire jusque dans la Capitainerie Générale du Guatemala grâce à la position d'interface occupée par la Louisiane et la Nouvelle-Orléans en particulier. Il importe donc pour les autorités espagnoles de le contrôler et de limiter son pouvoir de contagion, d'autant plus que la Nouvelle-Espagne se trouve en 1794 aux prises avec la crainte grandissante d'une conspiration révolutionnaire, comme le montre le dossier monté par l'Inquisition contre le Français Esteban Morel dès 1781 (Schifter, Aceves, Bret, 2011).

15 Conçue comme un glacis protecteur sur le plan géostratégique, la Louisiane espagnole apparaît paradoxalement comme une faille dans le dispositif de défense politicoreligieux. Sa position de carrefour atlantique et caribéen la place au cœur des circulations révolutionnaires mises en lumières par Jacques Godechot ou Annie Jourdan. Toutefois, dans ce même contexte des années 1790, et plus encore après 1793, il apparait que la Louisiane sous la domination du roi d'Espagne se trouve également au cœur de circulations contre-révolutionnaires.

Document 3 : Circulations révolutionnaires 


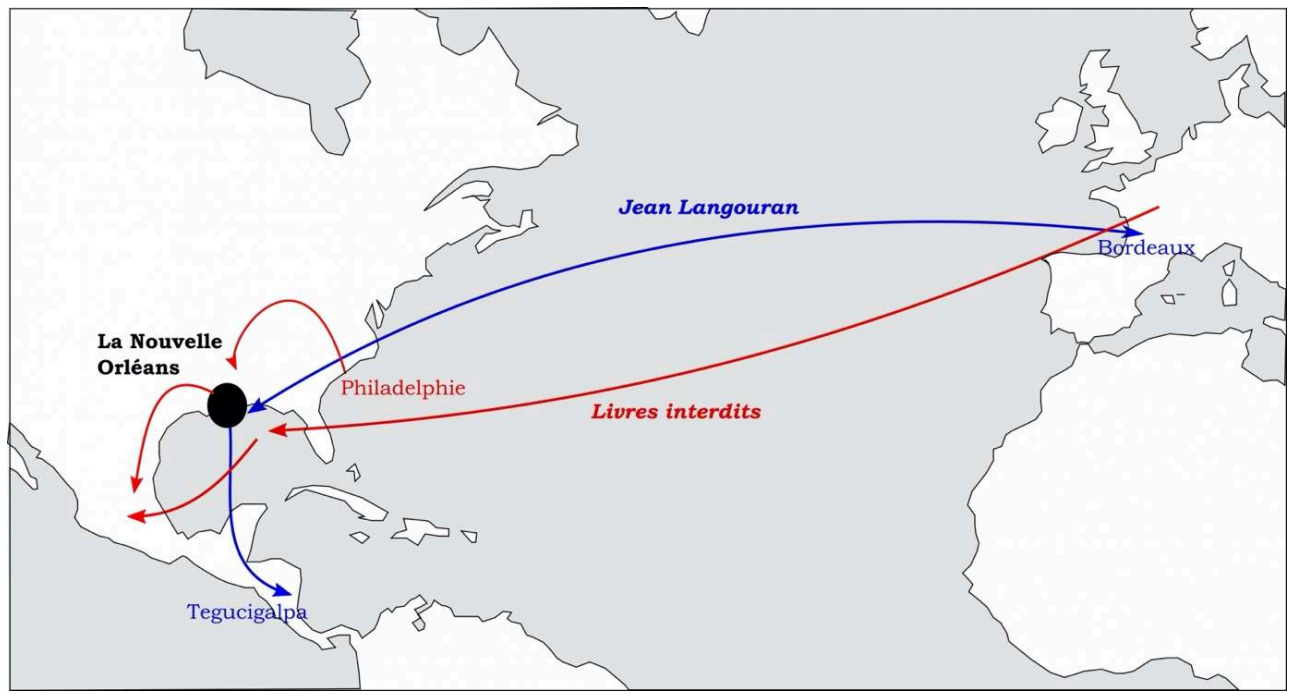

\section{Mobilités contre-révolutionnaires : trouver sa place dans la Caraïbe en révolution}

Si les possessions du roi d'Espagne en Amérique font figure de "citadelle assiégée " face aux mobilités révolutionnaires des deux côtés de l'Atlantique, il ne faut pas pour autant oublier qu'elles peuvent aussi constituer un véritable refuge pour les individus refusant l'évolution politique et sociale en Europe comme en Amérique. Les remous révolutionnaires engendrent dès 1789 des circulations contre-révolutionnaires à travers les itinéraires des exilés - aristocratie et prêtres réfractaires essentiellement qui se dirigent vers l'Espagne, l'Empire ou les îles britanniques. Dans le cadre caribéen, les propriétaires de Saint-Domingue cherchent refuge à Cuba - restée loyale à la Couronne alors que le continent se soulève au début du XIX ${ }^{e}$ siècle - ou aux États-Unis, répandant par leurs histoires la peur du « spectre » haïtien d'un soulèvement général des esclaves. Nathalie Dessens a ainsi étudié les flux des populations françaises de Saint-Domingue vers la Nouvelle-Orléans d'abord sous domination espagnole puis intégrée aux États-Unis, flux qui ont conduit à un renforcement du caractère français de la ville au XIXe siècle (Dessens, 2007). L'étude des demandes de passeport pour la Nouvelle-Orléans à partir des ports espagnols permet de reconstituer des itinéraires similaires pour comprendre les dynamiques des circulations contre-révolutionnaires autour de la Louisiane espagnole.

\section{A. France révolutionnaire ou Espagne monarchique?}

À ce titre, le cas de François Beauregard est particulièrement intéressant. À la suite des déboires qui le mènent de Marseille au port de Carthagène, il cherche à regagner la Nouvelle-Orléans par le premier bateau :

«très jeune il est passé depuis la ville de Marseille dans le royaume de France sa patrie à ces domaines où il est vecino, où il a établi sa maison, pour la gestion de son commerce ; mais ayant été obligé de collecter des dettes importantes dans la dite ville de Marseille, il décida de s'y rendre pour vérifier ce problème, arranger ses affaires et retourner à son domicile. C'est ce qu'il fit [en s'embarquant] sur le brigantin espagnol la Minerva, son capitaine et maître don Juan de Castañedo, en vertu de la licence du gouverneur de cette province [Louisiane] en conformité avec 
le témoignage joint ; mais à ce moment-là la Révolution éclata dans tout le royaume de France et comme le déclarant connaissait la mauvaise cause adoptée par cette injuste et tyrannique rébellion, in ne put s'empêcher de manifester son aversion pour un système si détestable, ce qui lui attira la haine et la persécution de la part des partisans locaux qui le traitèrent de traître car vassal et acquis à Votre Majesté Catholique à la Nouvelle-Orléans; et comme il avait appris qu'ils voulaient s'emparer de lui pour le punir et le tuer, il parvint à fuir en prenant la direction de Toulon où il se déclara ouvertement pour le parti des puissances alliées et quand celles-ci évacuèrent cette ville, le déclarant parvint à s'embarquer sur le navire de guerre espagnol le San Leandro du capitaine don Pedro de Carriazo sans avoir pu, dans la précipitation, mener quoi que ce soit d'autre ; et il arriva à Carthagène dans cette situation où il désembarqua après avoir été inscrit sur la liste des Emigrés et en tant que tel obtint le passeport correspondant... ${ }^{4}$.

19 Le récit de Beauregard permet tout d'abord de la projection atlantique et méditerranéenne d'un marchand né à Marseille et installé à la Nouvelle-Orléans. Grâce aux concessions d'Alejandro O'Reilly qui, à partir de 1769, ont constitué pour ainsi dire une feuille de route pragmatique pour ses successeurs, la Louisiane n'est pas intégrée à l'exclusif espagnol et conserve au contraire ses liens avec les réseaux commerciaux français qui se développent avec les encouragements d'une administration espagnole soucieuse de stimuler l'économie locale (Hoffman, 1992). L'obtention du statut de vecino $y$ del comercio de la Nouvelle-Orléans est en effet un avantage conséquent pour les marchands et autres aventuriers dans la mesure où une telle position permet de superposer les réseaux commerciaux hispaniques, français et anglo-américains. Dans le cas de Beauregard, on peut imaginer un marchand arrivé jeune à la Nouvelle-Orléans, ayant développé ses affaires entre Louisiane et Caraibe tout en gardant un lien avec les affaires familiales à Marseille. Par la suite, les troubles révolutionnaires de 1792-93 qui touchèrent la Provence précipitèrent un retour temporaire en France.

Une grande partie de la population française en Louisiane a manifesté une certaine sympathie pour la Révolution ainsi qu'un sentiment patriotique croissant dans les années 1780 et 1790. L'agitation révolutionnaire gagne la population française de Louisiane dès 1790 à travers l'envoi de pétitions pour obtenir un rattachement à « la mère patrie " (Havard et Vidal, 2008). La trajectoire de Beauregard prend le contre-pied du modèle dominant. Visiblement attaché à la royauté, il est menacé par les représailles qui suivent la reprise de Marseille avant de fuir à Toulon encore occupée par les Britanniques. Il se réfugie alors en Espagne en tant qu'émigré pour regagner la Nouvelle-Orléans. La succession des statuts invoqués par Beauregard dans sa demande de passeport mérite une attention particulière. Né à Marseille, Beauregard est manifestement de "nation française " si l'on reprend les catégories utilisées par Vanessa Mongey (Mongey, 2014). Opposé à la Révolution, il ne peut pas être « citoyen » et se retrouve donc exclu du corps social et politique de la France de la Convention : il figure ainsi sur la liste des «Émigrés ». Toutefois, il n'est pas un émigré « comme les autres » dans la mesure où il bénéficie également du statut de vecino de la NouvelleOrléans et partant du statut de sujet du roi d'Espagne. Beauregard a jonglé avec les différentes facettes d'une identité complexe et mouvante, comme bon nombre de Français et d'Anglo-Américains « à la fois capables et prêts à modifier leur identité et loyauté. La nationalité n'était qu'un composant du sens individuel de l'identité, et les individus peuvent considérer d'en changer volontairement pour de nombreuses raisons, habituellement connectées à l'espérance d'un gain ou intérêt personnel » (Smith et Hilton, 2010). Au final, l'évolution du contexte politique en France le 
contraint à se replier sur le seul statut de sujet de roi d'Espagne, ce qui se reflète à travers son itinéraire entre Amérique et Europe.

\section{B. Rester sujet du roi d'Espagne}

21 On retrouve une stratégie similaire dans le cas d'Alexandre Deblieux et Thérèse Isnard en 1804 :

« Alejandro Deblieux, vecino de la Nouvelle-Orléans, ancienne colonie espagnole, se présente de la meilleure manière pour exposer que, alors que la dite province reconnaissait le roi catholique comme souverain et grâce à la liberté dont jouissait cette ville (Nouvelle-Orléans), il s'embarqua pour le Havre de Grâce en territoire français après avoir obtenu le passeport correspondant du gouverneur de la Louisiane, qui était alors don Manuel de Salcedo (...). Après avoir conclu ses affaires dans cette ville, il désire rentrer comme bon espagnol avec sa femme et ses trois enfants en Floride occidentale à la première occasion opportune, en vertu de quoi... il supplie Votre Excellence d'infléchir la pitié du Roi pour qu'il concède le permis de passer à la dite province de Floride occidentale avec son épouse doña Teresa Francisca Isnard et leurs deux fils Alejandro Luis et Honorato, et leur fille Teresa Rosa, tous [trois] mineurs... $»^{5}$.

Ici, l'élément déclencheur de la démarche de loyauté de Deblieux et de sa famille est la vente de la Louisiane aux États-Unis en 1800 et la cession effective en 1803. Si l'on reprend la demande de passeport de Deblieux, il est parti de la Nouvelle-Orléans pour mener ses affaires au Havre. Comme Beauregard, c'est un marchand bénéficiant d'un double ancrage des deux côtés de l'Atlantique. Il obtient un premier passeport du gouverneur don Manuel de Salcedo, le dernier administrateur espagnol en Louisiane entre 1801 et 1803, avant le bref intermède de Laussat et la prise de contrôle par les Etats-Unis. Il est intéressant de s'arrêter sur la formulation utilisée : " alors que la dite province reconnaissait le roi catholique comme souverain et grâce à la liberté dont jouissait cette ville (Nouvelle-Orléans)». De fait, une telle affirmation de correspond pas à la réalité juridique de la Louisiane à ce moment-là. Celle-ci a été en effet cédée par l'Espagne à la France lors des négociations de paix avec Bonaparte. La condition était que la France conserverait la Louisiane comme colonie, de manière à conserver un rôle de protection de la Nouvelle-Espagne face aux appétits étatsuniens qui avaient remplacé les ambitions britanniques en Amérique du Nord (Havard et Vidal, 2008). Toutefois, le rêve américain de Bonaparte est bien éphémère et il accepte la proposition d'achat des États-Unis :

« Napoléon espère en tirer cinquante millions de francs or, finalement il en obtient quatre-vingts millions sur lesquels vingt sont retenus pour indemnisation des navires saisis. Cette vente surprenante, conclue le 3 mai 1803, intervient dans un contexte de montée des tensions avec l'Angleterre qui reprend les hostilités le 10 mai. » (Olivier, 2009)

Tout comme les Espagnols entre 1763 et 1765, les Français ne se sont guère pressés pour prendre possession de la colonie : c'est ainsi que l'Espagnol Manuel Salcedo assure la continuité du gouvernement, créant une impression confuse de continuité pour la population locale. Dans son expérience quotidienne, Alexandre Deblieux continue à traiter avec les autorités espagnoles et considère donc encore être sous l'autorité du roi d'Espagne. L'attachement manifesté par Deblieux dans sa requête reflète en outre la bienveillance que l'administration espagnole et son pragmatisme ont su susciter au sein des populations francophones et anglophones (Hilton, 2010). Lors de son voyage au 
Havre, la situation change drastiquement à la suite de la vente du 3 mai 1803. Deblieux, qui avait quitté ce qu'il semble considérer comme un territoire espagnol - du moins face à ses interlocuteurs espagnols, est confronté à la nécessité de retourner dans un territoire devenu étatsunien. Plutôt que de revenir à la Nouvelle-Orléans il sollicite un passeport pour la Floride Occidentale voisine (Alabama actuel). Plusieurs hypothèses peuvent être formulées pour essayer de comprendre une telle démarche. Le poids des réseaux commerciaux et l'insertion de Deblieux dans les réseaux caribéens espagnols et français est une première hypothèse. Il est également possible d'imaginer un positionnement politique royaliste qui lui ferait préférer le statut de sujet du roi d'Espagne à celui de citoyen des États-Unis. Il est enfin intéressant de noter que Deblieux s'affirme également de "nation espagnole » pour justifier sa démarche. Cela peut être une simple étiquette invoquée en fonction d'un objectif immédiat - rejoindre Pensacola. Cela peut être également une affirmation sincère reflétant une évolution identitaire complexe de certains Français de Louisiane espagnole au cours des événements des années 1790-1800. Ici, l'importance du concept de vecino utilisé par les deux hommes pour manifester leur loyauté peut expliquer leur volonté de regagner les territoires tenus par la Couronne espagnole. Tamar Herzog montre ainsi que «la domiciliation pouvait transformer les étrangers en natifs" (Herzog, 2011), que "l'appartenance aux communautés locales définissait les relations liant l'individu au royaume et que la 'loi de la domiciliation' était aussi importante, si ce n'est plus importante que la loi de la naissance (ius soli) and la loi de la descendance (ius sanguinis) » (Herzog, 2011). Les deux hommes ont d'autant plus de facilité à effectuer cette démarche que " pour les Espagnols du XVIII siècle [...] les individus avaient un 'droit naturel' à changer leur adhésion d'une communauté à une autre » (Herzog, 2011).

Document 4 : Circulations contre-révolutionnaires

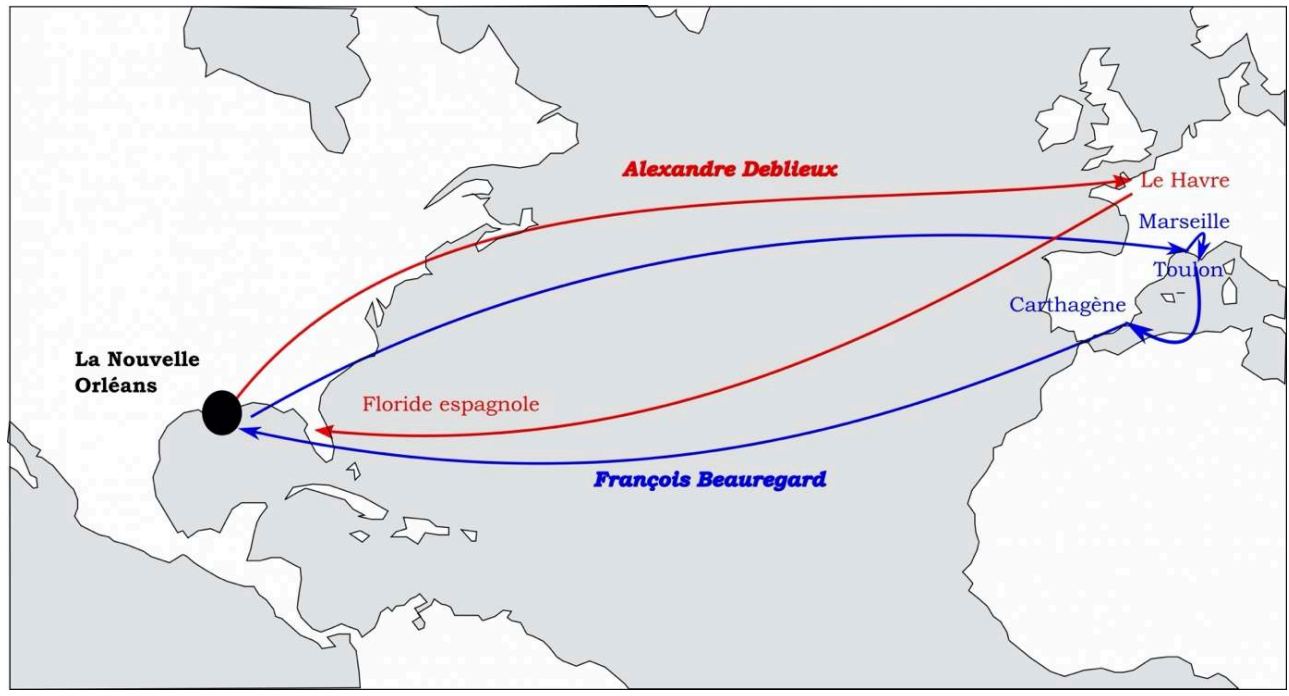

\section{Conclusion}

Expérience coloniale éphémère, la Louisiane espagnole constitue le creuset d'une société multiculturelle au sein de laquelle se croisent Français, Espagnols, AngloAméricains, Indiens, Esclaves et Noirs libres[19]. Ces mobilités se déploient le long du Mississippi, dans la Caraïbe, dans le monde atlantique et font de la Louisiane une région «à la croisée des empires ». Comme l'écrit Sylvia Hilton : 
« Entre 1763 et 1803, la vallée du Mississippi occupait le devant de la scène dans ce long processus historique décrit par Jeremy Alderman comme la 'lutte panAtlantique pour le contrôle mercantiliste, la loyauté politique, et finalement pour l'alliance militaire qui précipita la crise de l'Ancien Régime.' Une toile étroitement tissée d'intérêts interconnectés, processus et événements reliait la Louisiane espagnole aux vastes mondes atlantiques $»^{6}$.

Dans le contexte révolutionnaire des années 1790 , la circulation des hommes, des marchandises et des idées met en lumière différentes facettes de la Louisiane dans le dispositif impérial espagnol. Glacis protecteur, porte d'entrée, point de vulnérabilité, refuge, la région change de visage selon la perspective adoptée et les mobilités étudiées. On retrouve ici les caractéristiques d'une grande fluidité des hommes, des loyautés et des identités décrites aussi bien par Sylvia Hilton, Gene Allen Smith (Allen Smith et Hilton, 2010) que par Daniel Usner (Usner, 1992). Le brassage des populations permet ainsi l'élaboration de stratégies individuelles cherchant à mettre à profit les atouts d'une périphérie impériale à la centralité indéniable.

\section{BIBLIOGRAPHIE}

\section{Bibliographie}

ALLEN SMITH G. and HILTON S. L., 2010, Nexus of Empire: Negotiating Loyalty and Identity in the Revolutionary Borderlands, 1760s-1820s, University of Florida Press, 376 p.

ANDREU OCARIZ J. J., 1975, Luisiana española, Zaragoza, Talleres Editoriales Librería General, $85 \mathrm{p}$.

BENNASSAR B. (dir.), 1990, Les Français en Espagne à l'époque moderne (XVIe-XVIIIe siècles), Paris, CNRS, $258 \mathrm{p}$.

BURBANK J. et COOPER F., 2011, Empires. De la Chine ancienne à nos jours, Paris, Payot, 687 p.

CARDIM P., HERZOG T., RUIZ IBAÑEZ J. J. et SABATINI G. (eds.), 2012, Polycentric Monarchies: How did Early Modern Spain and Portugal Achieve and Maintain a Global Hegemony?, Eastbourne, Sussex Academic Press, 320 p.

DARNTON R., 1991, Edition et sédition. L'univers de la littérature clandestine au XVIIIe siècle, Paris, Gallimard, $288 \mathrm{p}$.

DAWDY S. L., 2007, « La Nouvelle-Orléans au XVIIIe siècle Courants d'échange dans le monde caraïbe », Annales. Histoire, Sciences Sociales, 2007/3 62e année, p. 663-685.

DESSENS N., 2007, From Saint-Domingue to New Orleans. Migration and Influences, Gainesville, FL : University of Florida Press, $257 \mathrm{p}$.

GODECHOT J., 1986, Les Révolutions, Paris, PUF, Collection Nouvelle Clio, 448 p.

GÓMEZ A., 2017, Le Spectre de la Révolution Noire. L'impact de la révolution haïtienne dans le monde atlantique, 1790-1886, Rennes, PUR, 318 p. 
HAVARD G. et VIDAL C., 2008, Histoire de l'Amérique française, Paris, Flammarion, Collection Champs Histoire, $863 \mathrm{p}$.

HERZOG T., 2003, Defining Nations. Immigrants and Citizens in Early Modern Spain and Spanish America, New Haven and London, Yale University Press, 334 p.

HILTON S., 2014, "Spanish Louisiana in Atlantic Contexts: Nexus of Imperial Transactions and International Relations" dans Vidal C., Louisiana: Crossroads of the Atlantic World, Philadelphia, University of Pennsylvania Press, p. 68-85.

HILTON S., 2010, « Loyalty and Patriotism on North American Frontiers: Being and Becoming Spanish in the Mississippi Valley, 1776-1803. » dans Allen Smith G. and Hilton S. L., Nexus of Empire: Negotiating Loyalty and Identity in the Revolutionary Borderlands, 1760s-1820s, University of Florida Press, p. 8-36.

HOFFMAN P., 1992, Luisiana, Madrid, Editorial MAPFRE, 328 p.

JIMÉNEZ A., 2006, El Gran Norte de México. Una frontera imperial en la Nueva España (1540-1820), Madrid, Editorial Tébar, 536 p.

JOURDAN A., 2004, La Révolution, une exception française ?, Paris, Champs Flammarion, 461 p.

LAUSSAT, P. C., Mémoires sur ma vie, pendant les années 1803 et suivantes, Pau, B. Hauvette (dir.), imprimerie des Gaves, 2001, réédition, 3 tomes.

LOMNÉ G., 2011, « 1794, ou l'année de la « sourde rumeur », la faillite de l'absolutisme éclairé dans la vice-royauté de Nouvelle-Grenade » dans Annales de la Révolution française, Juilletseptembre 2011, p. 9-29.

MARICHAL C. et SOUTO M., 2012, "La Nueva España y el financiamiento del Imperio Español en América : los situados para el Caribe en el siglo XVIII" dans Marichal C. et Grafenstein J. von, El secreto del Imperio español : los situados coloniales en el siglo XVIII, México, Colegio de México, p. 61-94.

MONGEY V., 2009, « Les vagabonds de la république : les révolutionnaires européens aux Amériques, 1780-1820 » dans Clément Thibaud, Francesca Morelli and Geneviève Verdo (eds.), Les empires atlantiques des Lumières au libéralisme (1763-1865), Rennes : Presses Universitaires de Rennes, p. 67-82.

MONGEY V., 2014, « 'Des Français indignes de ce nom' : Être et rester Français en Louisiane (1803-1830) » dans Cécile Vidal, Être et se revendiquer Français dans le monde atlantique. Nation, empire, et race (XVIe-XIXe siècle), Paris, Edition EHESS, p. 187-208.

OLIVIER J.-M., 2009, « Bernadotte, Bonaparte et la Louisiane » dans Christophe Belaubre, Jordana Dym et John Savage (dir.), Napoléon et les Amériques, Toulouse, PUM, Collection Méridiennes.

NARRETT D., 2014, Adventurism and Empire: the Struggle for Mastery in the Louisiana-Florida Borderlands, 1762-1803, Chapel Hill, University of North Carolina Press, 392p.

SCHIFTER L., ACEVES P. et BRET P., 2011, « L'Inquisition face aux Lumières et à la révolution française en Nouvelle-Espagne : le dossier et le procès d'Esteban Morel (1781-1795) », Annales historiques de la Révolution française, 2011, 365, p. 103-127.

TERRIEN N., 2015 , » Des patriotes sans patrie » Histoire des corsaires insurgés de l'Amérique espagnole (1810-1825), Mordelles, Les Perséides, 381p.

USNER D., 1992, Indians, Settlers, and Slaves in a Frontier Exchange Economy: The Lower Mississippi Valley Before 1783, Chapel Hill, University of North Carolina Press, 314p. 
VIDAL C., 2014, Louisiana: Crossroads of the Atlantic World, Philadelphia, University of Pennsylvania Press, $304 \mathrm{p}$.

VIDAL C., 2014, Être et se revendiquer Français dans le monde atlantique. Nation, empire, et race (XVIe-XIXe siècle), Paris, Edition EHESS, $270 \mathrm{p}$.

\section{NOTES}

1. Expression utilisée lors de la révolte de 1768.

2. AGN, Inquisición, 1389, Exp22, Expediente formado con el edicto publicado en esta corte en 26 de octubre de 1794 prohibiendo el libro titulado El Desengaño del Hombre

3. AGI, CUBA, 163A, Testimonio de las ultimas diligencias en el lance ocurrido entre el Capitán del Regimiento de Luisiana D. José LeBlanc y D. Arturo Morgan, f. 648r.

4. AGI, INDIFERENTE, 2120, N.18.

5. AGI, INDIFERENTE, 2135, N.85.

6. Allen Smith, Gene et Hilton, Sylvia L., Nexus of Empire. Negotiating Loyalty and Identity in the Revolutionary Borderlands, 1760's - 1820's, University Press of Florida, Tallahassee, p.4.

\section{RÉSUMÉS}

La présence espagnole en Louisiane entre 1763 et 1803 a placé la région à la croisée des empires espagnol, français et anglo-américain. Dans le contexte des révolutions atlantiques, la région est de facto placée au cœur d'une intense circulation des hommes et des idées, tout en demeurant un territoire périphérique aux confins des empires, un véritable angle mort qui autorise les circulations clandestines tout comme la plasticité des loyautés. Originellement conçue comme un glacis protecteur censé protéger la Nouvelle-Espagne des appétits anglo-américains, la Louisiane s'avère en fait être un espace vulnérable à la pénétration des idées révolutionnaires en raison de sa connexion aux réseaux français comme hispaniques. A l'opposé, la permanence de la monarchie espagnole jusqu'en 1803 génère des itinéraires politiques et identitaires contrerévolutionnaires à travers l'Atlantique.

The Spanish presence in Louisiana between 1763 and 1803 placed the region on the crossroads of the Spanish, French, British and later on American empires. In the context of the Atlantic Revolutions, the region was at the heart of intense circulations of individuals and ideas. It remained however a periphery of these empires, allowing clandestine mobility accompanied by changing loyalties. Initially thought by the Spaniards as a barrier against feared anglo-american expansion, Louisiana appeared as open to revolutionary ideas because of her old connections both to the French and the Hispanic world. But governed by a monarchical regime until 1803, Luisiana was also at the heart of counter-revolutionary mobility between both shores of the Atlantic 
INDEX

Mots-clés : Louisiane espagnole, Révolutions atlantiques, Mobilités, XVIIIe siècle, Identités

Keywords : Spanish Louisiana, Atlantic Revolutions, Mobilities, 18th century, Identities

\section{AUTEUR}

\section{SOIZIC CROGUENNEC}

MCF Histoire moderne, MINEA EA7485, Université de Guyane 